\title{
Predictors of poor function in RA based on two prospective UK inception cohorts. Do comorbidities matter?
}

\author{
Amanda D. Busby (1) 1, James Wason ${ }^{2}$, Arthur G. Pratt ${ }^{3,4}$, Adam Young1, \\ John D. Isaacs ${ }^{3,4}$ and Elena Nikiphorou ${ }^{5,6}$
}

\begin{abstract}
Objectives. Evidence suggests that factors beyond disease activity associate with functional disability in RA. The primary study objective was to explore associations between comorbidities, sociodemographic factors and functional outcomes at five and 10 years.

Methods. RA patients from two UK prospective cohorts were grouped into low $(<1.5)$ and high $(\geq 1.5)$ five- and 10-year health assessment questionnaire (HAQ) score. Clinical variables (e.g. disease activity, rheumatoid nodules, erosions) and sociodemographic factors (e.g. ethnicity, deprivation) were recorded at baseline and yearly thereafter. Comorbidity was measured using the Rheumatic Diseases Comorbidity Index (RDCl). Binary logistic regression models were fitted using multiple imputation.

Results. In total, 2701 RA patients were recruited (mean age 56.1 years, $66.9 \%$ female). A total of 1718 (63.4\%) had five-year and 820 (30.4\%) 10-year follow-up data. In multivariable analysis, no association was found between $\mathrm{RDCl}$ and $\mathrm{HAQ} \geq 1.5$ at five or 10 years. Sociodemographic factors (increased age at disease onset, female gender, minority ethnicity) were associated with higher odds of $H A Q \geq 1.5$ at five and 10 years, with worse deprivation additionally associated with $\mathrm{HAQ} \geq 1.5$ at 10 years (OR $0.79,95 \% \mathrm{Cl}: 0.69,0.90)$.

Conclusion. Comorbidities at baseline have not been found to be associated with worse RA functional outcome in the long-term. On the other hand, sociodemographic factors, independently of disease measures, are associated with worse functional outcome in RA at five and 10 years, in models adjusting for comorbidity burden. Tailoring management interventions according to not only clinical disease parameters but also patient sociodemographic factors may improve long-term outcomes including functional disability.
\end{abstract}

Key words: sociodemographic factors, comorbidity, multimorbidity, Health Assessment Questionnaire, rheumatoid arthritis

\section{Rheumatology key messages}

- Sociodemographic factors, independently of disease activity, are associated with worse functional outcome in RA. - Identifying patients at increased risk of functional disability at initial review enables targeted management.

\section{Introduction}

Functional disability in RA is a well-recognised and historically reported debilitating outcome of disease,

\footnotetext{
${ }^{1}$ Centre for Health Services and Clinical Research, Life and Medical Sciences, University of Hertfordshire, Hatfield, ${ }^{2}$ Population Health

Sciences Institute, ${ }^{3}$ Translational and Clinical Research Institute,

Faculty of Medical Sciences, Newcastle University,

${ }^{4}$ Musculoskeletal Unit, Newcastle upon Tyne Hospitals NHS

Foundation Trust, Freeman Hospital, Newcastle upon Tyne, ${ }^{5}$ Centre

for Rheumatic Diseases, King's College London and ${ }^{6}$ Rheumatology

Department, King's College Hospital, London, UK

Submitted 12 May 2021; accepted 20 July 2021
}

adding significantly to the overall global burden of RA [1]. Worsening functional ability in RA has been linked to the ongoing, progressive course of this chronic, inflammatory musculoskeletal condition and comes at both physical and psychological cost to patients. Indeed,

Correspondence to: Amanda D. Busby, Centre for Health Services and Clinical Research, Life and Medical Sciences, University of Hertfordshire, College Lane, Hatfield AL10 9AB, UK.

E-mail: a.busby@herts.ac.uk 
functional measures are often used as a substitute for quality of life in RA [2].

Generally, it is accepted that poor disease control negatively impacts functional ability, especially in early disease, but some studies have shown this is not always the case. Functional deterioration has been reported in $10-21 \%$ of early RA patients with moderate disease activity when following standard treatment [3]. Beyond inflammation, other factors that may cause worse function need to be considered.

The comorbidity burden in RA is substantial, even on first presentation of disease [4]. Yet, this observation does not seem to be accompanied by increasing disease severity, whether measured by markers of high disease activity or worse physical function [4]. Additionally, the presence of comorbidities has been associated with unfavourable functional and disease activity outcomes over two years, despite intensive treatment strategies aiming for remission [5]. Disentangling the underlying mechanisms and understanding potential causal links between comorbidities and disease outcomes including function in RA is challenging. Associations between comorbidities and poor health outcomes including physical disability have been traditionally studied alongside other disease-related factors, e.g. inflammation, but not in relation to non-biological, sociodemographic factors.

Sociodemographic determinants of health play a crucial role in health and disease and have gained much attention in recent times, where there is an expectation to manage patients more holistically, with care tailored to their individual physical and psychosocial needs. To achieve this, we have argued for a better understanding of the role of sociodemographic factors, alongside important clinical factors including comorbidities, as drivers of adverse outcomes in RA [6]. In seeking to unravel these so-called 'syndemic' elements [6], this study stems from a larger research initiative to understand multimorbidity in the disease and risk-stratify patients according to individual need .

The aim was to identify predictors of worse five- and 10-year functional outcome, measured using the $\mathrm{HAQ}$, using information available at first outpatient appointment. Of particular interest was whether patients affected by comorbidities have an elevated risk of poor functional ability, taking into account sociodemographic factors.

\section{Methods}

\section{Study population}

This study used data collected within the Early RA Study (ERAS, 1986-2001) and Early RA Network (ERAN, 20022012). These are consecutive prospective cohorts, designed similarly regarding recruitment, variables collected and follow-up timings, often studied together and previously described in detail [7]. ERAS patients were recruited from nine hospitals in England and ERAN patients from 23 centres across England, Wales and Northern Ireland. Dates of deaths occurring in the UK were reported by NHS Digital for all participants who consented to follow-up. ERAS patients gave informed consent as required at time of enrolment [prior to Good Clinical Practice (GCP) implementation], approved by the East Hertfordshire Local Research Ethics Committee. ERAN patients gave informed, written consent, approved by the Trent Research Ethics Committee .

\section{Baseline measures}

\section{Comorbidities}

The variable of interest was baseline comorbidity, measured using the Rheumatic Disease Comorbidity Index (RDCl [8]). Scores ranged from 0 to 9 , where higher scores indicated a higher comorbidity burden, and were used as a continuous variable in the analysis. The RDCl arranges 11 illness types into eight categories (lung disease, heart attack/other cardiovascular/stroke), fracture, depression, cancer, ulcer/stomach problem, hypertension, diabetes), defined using International Classification of Diseases, Ninth Revision, Clinical Modification (ICD-9$\mathrm{CM}$ ) codes. As well as overall $\mathrm{RDCl}$ values, individual disease categories were assessed to determine association with high HAQ outcome.

\section{Sociodemographic and clinical variables}

At baseline, standard demographic data were recorded including age at onset, gender and ethnicity. Patient postcodes were used to derive an Index of Multiple Deprivation [9] (IMD), Welsh Index of Multiple Deprivation [10] (WIMD) or Northern Ireland Multiple Deprivation Measure [11] (NIMDM) rank, then grouped into quintiles to enable comparison across regions. Clinical data collected at each appointment included $\mathrm{HAQ}$, BMI, haemoglobin, ESR and presence of erosions, rheumatoid nodules, RF and anti-cyclic citrullinated peptide autoantibodies (anti-CCP). Disease activity was measured using the 28-joint Disease Activity Score DAS28 [12] for ERAN and the Disease Activity Score (DAS [13]) in the ERAS cohort. DAS scores were transformed so they could be directly comparable to the DAS28 using a formula previously tested and applied on the same data (Carpenter et al. [14]). Months from onset of symptoms to first rheumatology outpatient appointment and from first appointment to prescription of the first DMARD were each recorded. Variables were continuous (e.g. age, haemoglobin, ESR) or categorical, e.g. IMD (quintiles; 1, most deprived to 5, least deprived), smoking status (ever/ never), serostatus (positive/negative RF and/or anti-CCP), ethnicity (white/minority ethnic).

\section{Outcome measures}

Five-year and 10-year HAQ

$\mathrm{HAQ}$ scores above 1.5 indicate considerable levels of disability, shown to be the value above which progressive damage to large joints (shoulder, elbow, hip, knee, ankle) is expected to increase [15]. Therefore, the primary outcome of interest in this analysis was HAQ score studied as a binary outcome to indicate high $(\geq 1.5)$ vs low $(<1.5)$ 
$\mathrm{HAQ}$ at five years. $\mathrm{HAQ}$ at 10 years was investigated in secondary analysis.

\section{Statistical analysis}

\section{Five-year and 10-year HAQ}

The statistical analysis was prespecified (Supplementary Data S1, available at Rheumatology online) for HAQ outcomes. Descriptive statistics were calculated for patients with $H A Q<1.5$ and $H A Q \geq 1.5$ values at five and 10 years; medians and interquartile ranges (IQR) for continuous variables, frequencies and proportions for binary/ordinal. Odds ratios comparing the groups were obtained using binary logistic regression models for each covariate under consideration, fitting separate models for five- and 10-year HAQ. Five-year outcomes were not used in 10-year models, because only baseline factors were of interest. Variables with a likelihood ratio test $P$-value $<0.2$ were tested in multivariable analysis.

Models for five-year and 10-year HAQ were built using forward selection based on clinical relevance and statistical robustness to obtain odds ratios (ORs) and 95\% Cls. Age at onset, gender and RDCl were included a priori. Interaction testing was undertaken as appropriate to determine inclusion of interaction terms and is described in detail in Supplementary Data S2, available at Rheumatology online. Once model building was complete, multicollinearity between covariates was assessed, excluding variables with centred variance inflation factors $>10$. After models were determined using complete cases, multiple imputation by chained equations was used. All baseline variables in the complete case model and HAQ outcomes were imputed. Baseline and five-year values of clinical covariates (measured annually) were included in the imputation model for the five-year HAQ model, similarly baseline and 10-year values in the 10-year HAQ model, if available. Patients who had died before year five/10, respectively, were excluded from imputation models. Each model was performed with 20 imputations. Continuous variables were modelled using linear regression, binary using logistic regression and ordinal using predictive mean matching.

Although two-sided $P$-values of $<0.05$ are referred to as significant, a multiple testing correction was not applied. Thus, $P$-values should be interpreted with caution and significant results seen as hypothesis-generating rather than confirmatory.

$H A Q$ sensitivity analyses

In these analyses, five- and 10-year HAQ were used in their continuous form. In addition, a binary $\mathrm{RDCl}$ variable was formed, comparing patients with no comorbidities $(\mathrm{RDCl}=0)$ to those with at least one $(\mathrm{RDCl} \geq 1)$.

Supplementary analyses: Short-Form 36 health questionnaire

To support the HAQ findings, the effect of baseline comorbidity burden on health-related quality of life (HRQoL) at five years was assessed, using the ShortForm 36 Health Questionnaire (SF-36). Full details of the methods used can be found in Supplementary Data S2, available at Rheumatology online.

\section{Results}

The ERAS and ERAN cohorts recruited 2701 patients, mean age 56.1 years and $66.9 \%$ female. Median time from onset to first outpatient appointment was 6 months. By year five, 1718 (63.4\%) patients remained enrolled, $185(6.8 \%)$ were known to have died and a further 798 $(29.5 \%)$ were no longer in follow-up. At year 10, 820 (30.4\%) were enrolled, 454 (16.8\%) had died and 1427 $(52.8 \%)$ were not in follow-up. Comparisons between those still enrolled and those no longer followed up are shown in Supplementary Tables S1 and S2, available at Rheumatology online. Those remaining enrolled at five years had median age of onset of 55.5 years (IQR 4565) compared with 62 (IQR 50-70) years for those no longer followed up.

\section{Five-year HAQ}

Table 1 summarises baseline characteristics for patients with $\mathrm{HAQ}<1.5$ and $\mathrm{HAQ} \geq 1.5$ at five years, with scores available for 1441 (83.9\%) of those still in follow-up. A higher age of onset was seen among the $H A Q \geq 1.5$ group (median 58, IQR 48-68 compared with median 55, IQR 43-63). A larger proportion of females had $\mathrm{HAQ} \geq 1.5$ (75.3\% compared with $63.9 \%$ for $\mathrm{HAQ}<1.5)$. More patients had a minority ethnic background with $\mathrm{HAQ} \geq 1.5$ (4.7\% compared with $1.7 \%$ ), although just 37 patients identified as belonging to a minority ethnic group. A larger proportion of patients with $\mathrm{HAQ} \geq 1.5$ were in the most deprived IMD quintile $(15.8 \%$ compared with $11.0 \%$ ). Conversely, $28.8 \%$ of patients with $\mathrm{HAQ}<1.5$ were in the least deprived group compared with $20.2 \%$ with $\mathrm{HAQ} \geq 1.5$.

More patients with $\mathrm{HAQ} \geq 1.5$ had at least one comorbidity (with $18.2 \%$ having an $\mathrm{RDCl}$ score of 1 and $11.8 \%$ scoring $\geq 2$, compared with the $\mathrm{HAQ}<1.5$ group, where $13.5 \%$ had RDCl score of 1 and $9.9 \%$ had a score of $\geq 2$ ). The median RDCl score for both groups was 0 , although the IQR was slightly wider for $H A Q \geq 1.5$ group. Each comorbidity category was slightly more prevalent among the $H A Q \geq 1.5$ group. Hypertension was the most common comorbidity, present in $9.0 \%$ of patients with $\mathrm{HAQ}<1.5$ and $12.2 \%$ of patients with $H A Q \geq 1.5$. Baseline $H A Q$ was higher for those with high five-year HAQ (median 1.5, IQR 1.002.13 compared with 0.88 , IQR $0.38-1.38$ ) and DAS was similarly elevated (5.30, IQR 4.47-6.08 compared with 4.51, IQR 3.68-5.49).

Results of univariable analyses are shown in Supplementary Table S3, available at Rheumatology online. Increased age at onset, being female, or of minority ethnicity were associated with high $\mathrm{HAQ}$, with $P<0.2$. The two least deprived IMD quintiles were associated with a reduction in odds of $H A Q \geq 1.5$. Several clinical and treatment variables were associated with 
TABLE 1 Patient, comorbidity and disease characteristics according to elevated HAQ status

Five-year $H A Q<1.5 \quad$ Five-year $H A Q \geq 1.5 \quad$ Ten-year $H A Q<1.5 \quad$ Ten-year $H A Q \geq 1.5$

\begin{tabular}{|c|c|c|c|c|}
\hline Total $n(\%)$ & 1007 (69.9) & $434(30.1)$ & 438 (60.9) & 281 (39.1) \\
\hline \multicolumn{5}{|l|}{ Sociodemographic } \\
\hline $\begin{array}{l}\text { Age at onset (years): median } \\
\text { (IQR) }\end{array}$ & $55(45-63)$ & $58(48-68)$ & $52(43-61)$ & $55(45-64)$ \\
\hline Female: $n(\%)$ & $643(63.9)$ & $327(75.3)$ & $280(63.9)$ & $215(76.5)$ \\
\hline White ethnicity: $n(\%)$ & 982 (98.3) & $410(95.3)$ & $426(98.6)$ & $263(94.9)$ \\
\hline Ever smoked: $n(\%)$ & $394(48.1)$ & $177(50.1)$ & 165 (41.9) & $110(44.0)$ \\
\hline \multicolumn{5}{|l|}{ IMD quintiles: $n(\%)$} \\
\hline 1 (Most deprived) & $97(11.0)$ & $61(15.8)$ & $40(9.7)$ & $57(20.7)$ \\
\hline 2 & $123(14.0)$ & $68(17.6)$ & $48(11.7)$ & $50(18.1)$ \\
\hline 3 & $211(24.0)$ & $101(26.2)$ & $87(21.1)$ & 64 (23.2) \\
\hline 4 & $196(22.3)$ & $78(20.2)$ & $91(22.1)$ & $43(15.6)$ \\
\hline 5 (Least deprived) & $253(28.8)$ & $78(20.2)$ & $146(35.4)$ & $62(22.5)$ \\
\hline \multicolumn{5}{|l|}{ Comorbidity } \\
\hline $\begin{array}{l}\text { RDCl (continuous): Median } \\
\text { (IQR) }\end{array}$ & $0(0-0)$ & $0(0-1)$ & $0(0-0)$ & $0(0-0)$ \\
\hline Lung disease: $n(\%)$ & $58(5.8)$ & $28(6.5)$ & $22(5.0)$ & $13(4.6)$ \\
\hline $\begin{array}{l}\text { Heart attack, other CV, } \\
\text { stroke: } n(\%)\end{array}$ & $44(4.4)$ & $25(5.8)$ & $12(2.7)$ & $3(1.1)$ \\
\hline Fractures: $n(\%)$ & $3(0.3)$ & $2(0.5)$ & $2(0.5)$ & $1(0.4)$ \\
\hline Depression: $n(\%)$ & $15(1.5)$ & $10(2.3)$ & $4(0.9)$ & $6(2.1)$ \\
\hline Cancer: $n(\%)$ & $13(1.3)$ & $10(2.3)$ & $4(0.9)$ & $4(1.4)$ \\
\hline $\begin{array}{l}\text { Ulcer or stomach problem: } n \\
(\%)\end{array}$ & $24(2.4)$ & $18(4.1)$ & $8(1.8)$ & $8(2.8)$ \\
\hline Hypertension: $n(\%)$ & $91(9.0)$ & $53(12.2)$ & $24(5.5)$ & $17(6.0)$ \\
\hline Diabetes: $n(\%)$ & $16(1.6)$ & $9(2.1)$ & $8(1.8)$ & $6(2.1)$ \\
\hline \multicolumn{5}{|l|}{ Clinical measures } \\
\hline \multirow{2}{*}{$\begin{array}{l}\text { Onset to 1st appt: median } \\
\text { (IQR) }\end{array}$} & $6(4-12)$ & $7(4-12)$ & $7(4-12)$ & $6(4-12)$ \\
\hline & $n=1002$ & $n=431$ & $n=438$ & $n=281$ \\
\hline \multirow{2}{*}{$\begin{array}{l}\text { 1st appt to 1st DMARD: me- } \\
\text { dian (IQR) }\end{array}$} & $2(0-4)$ & $1(0-4)$ & $2(1-8)$ & $2(1-6)$ \\
\hline & $n=894$ & $n=415$ & $n=378$ & $n=268$ \\
\hline HAQ: median (IQR) & $\begin{array}{c}0.88(0.38-1.38) \\
n=998\end{array}$ & $\begin{array}{c}1.50(1.00-2.13) \\
n=428\end{array}$ & $\begin{array}{c}0.75(0.25-1.25) \\
n=438\end{array}$ & $\begin{array}{c}1.38(0.88-2.00) \\
n=281\end{array}$ \\
\hline \multirow[t]{2}{*}{ DAS28: median (IQR) } & $4.51(3.68-5.49)$ & $5.30(4.47-6.08)$ & $4.46(3.60-5.29)$ & $5.11(4.30-5.93)$ \\
\hline & $n=988$ & $n=427$ & $n=433$ & $n=279$ \\
\hline BMI $\left(\mathrm{kg} / \mathrm{m}^{2}\right)$ : median (IQR) & $\begin{array}{c}25.40(22.80-28.40) \\
n=920\end{array}$ & $\begin{array}{c}26.20(23.05-29.76) \\
n=397\end{array}$ & $\begin{array}{c}25.0(22.6-27.4) \\
n=400\end{array}$ & $\begin{array}{c}25.2(22.5-28.7) \\
n=251\end{array}$ \\
\hline Erosions: $n(\%)$ & $251(25.5)$ & $120(28.2)$ & $92(21.2)$ & $75(27.6)$ \\
\hline Nodules: $n(\%)$ & $62(6.2)$ & $26(6.0)$ & $24(5.5)$ & $22(7.8)$ \\
\hline Haemoglobin (g/dl): median & $12.8(11.8-13.8)$ & $12.6(11.7-13.8)$ & $12.8(11.9-13.8)$ & $12.5(11.4-13.5)$ \\
\hline (IQR) & $n=1001$ & $n=427$ & $n=434$ & $n=279$ \\
\hline Seropositive: $n(\%)$ & $702(73.4)$ & 327 (77.9) & $315(73.3)$ & $209(76.0)$ \\
\hline \multirow[t]{2}{*}{ ESR (mm/h): median (IQR) } & $30(15-54)$ & $36(18-60)$ & $30(14-50)$ & $40(24-64)$ \\
\hline & $n=956$ & $n=416$ & $n=430$ & $n=279$ \\
\hline
\end{tabular}

1st appt to 1st DMARD: time (months) from first appointment to starting DMARD; DAS28: Disease Activity Score (28 count); IMD: Index of Multiple Deprivation; Onset to 1st appt: time (months) from symptom onset to first outpatient appointment; RDCl: Rheumatic Diseases Comorbidity Index.

$\mathrm{HAQ} \geq 1.5$, with $P<0.2$. Notably, increasing baseline $\mathrm{HAQ}$ was associated with a large increase in odds of high five-year HAQ.

\section{Multivariable analyses}

Results of multivariable analyses are shown in Table 2. No association was found between baseline $\mathrm{RDCl}$ and $\mathrm{HAQ} \geq 1.5$ at five years in models fully adjusted for all relevant covariates (OR 1.06, 95\% Cl: 0.90, 1.26).
Patients of minority ethnicity were at higher risk of high five-year $\mathrm{HAQ}$, but with a wide $\mathrm{Cl}$ (OR 4.29, 95\% Cl: $2.13,8.64)$. A strong association between baseline $\mathrm{HAQ}$ and five-year $\mathrm{HAQ}$ was seen (OR 3.62, 95\% Cl: 3.01 , 4.35). Increased age at onset, female gender, increased onset to first outpatient time and seropositive status at baseline were all associated with five-year $\mathrm{HAQ} \geq 1.5$ scores. Worse deprivation levels were associated with increased $H A Q$ values, but including ethnicity attenuated 
TABLE 2 Sociodemographic and clinical factors associated with HAQ outcomes

5-year HAQ $(n=2516)$

Odds ratio $(95 \% \mathrm{Cl})$

$1.06(0.90,1.26)^{*}$

$1.02(1.01,1.03)$

$1.48(1.11,1.97)$

$3.62(3.01,4.35)$

$4.29(2.13,8.64)$

$1.03(1.01,1.05)$

$0.95(0.85,1.05)^{* *}$

$1.67(1.21,2.30)$

$0.99(0.99,1.00)$ 10-year HAQ $(n=2247)$

Odds ratio $(95 \% \mathrm{Cl})$

$0.94(0.73,1.22)^{*}$

$1.02(1.00,1.03)$

$1.58(1.10,2.25)$

$2.47(1.80,3.41)$

$3.65(1.55,8.59)$

$0.79(0.69,0.90)$

a

$\mathrm{ESR}(\mathrm{mm} / \mathrm{h})$

Variables tested in univariable analysis shown in Supplementary Table S2, available at Rheumatology online. IMD: index of multiple deprivation; RDCl: rheumatic diseases comorbidity index. Variables tested in multivariable analyses: Five-year $H A Q$ : RDCl, age at onset, gender, $\mathrm{HAQ}$, disease activity score, seropositive status, haemoglobin, ethnicity, IMD, time (months) from symptom onset to first outpatient appointment, time (months) from first appointment to commencing DMARD, BMI, ESR. Ten-year HAQ: RDCl and each component individually, age at onset, gender, HAQ, disease activity score, presence of erosions, BMI, haemoglobin, ethnicity, IMD, time (months) from first appointment to commencing DMARD. ${ }^{a}$ Not selected in univariable analysis $(P>0.2)$. b Not selected in multivariable analysis $(P>0.05)$. Multivariable logistic regression models; gender, age at onset and $\mathrm{RDCl}$ included a priori. ${ }^{*} P>0.05$ (not significant). ${ }^{* *} \mathrm{All} P<0.02$ except IMD $(P=0.316)$ at five years.

this association, suggesting a potential confounding effect. Evidence of association with ESR and odds of high $\mathrm{HAQ}$ was found but with an extremely small effect size.

\section{0-year HAQ}

Table 1 compares sociodemographic, comorbidity and disease characteristics, with $\mathrm{HAQ}$ scores recorded for $719(87.7 \%)$ of those in follow up at year 10 . Patients with 10-year $\mathrm{HAQ} \geq 1.5$ scores were more likely to be slightly older (median 55, IQR 45-64 compared with median 52, IQR 43-61), female (76.5\% v 63.9\%) and of minority ethnic origin $(94.9 \% \mathrm{v} 98.6 \%)$. Differences in deprivation were more marked than at five years, $35.4 \%$ of patients with $\mathrm{HAQ}<1.5$ compared with just $22.5 \%$ of those with $\mathrm{HAQ} \geq 1.5$ were in the least deprived quintile. No notable differences were seen in RDCl or any comorbidity component. Again, baseline HAQ was higher for those with high 10-year HAQ (median 1.38, IQR 0.88-2.00 compared with 0.75, IQR 0.25-1.25) and similarly for baseline DAS28 (median 5.11, IQR 4.305.93 compared with 4.46, IQR 3.60-5.29). More patients with high 10 -year HAQ scores had erosions $(27.6 \%$ vs $21.2 \%)$ and nodules $(7.8 \%$ vs $5.5 \%)$ at baseline.

Results of univariable analysis are shown in Supplementary Table S3, available at Rheumatology online. As with five-year $H A Q$, increased age at onset, female gender, minority ethnicity and more deprived IMD quintiles were individually associated with high 10-year HAQ scores, shown in Table 2. Baseline RDCl was not associated with high 10-year HAQ scores, in contrast with five-year HAQ. Among baseline clinical measures, first appointment to first DMARD time, baseline $H A Q$, baseline DAS28, BMI, haemoglobin and ESR remained associated with $P<0.2$. Although associated with fiveyear $\mathrm{HAQ}$, onset to first appointment time and being seropositive were not associated with 10-year HAQ. Conversely, presence of erosions at baseline were associated with $\mathrm{HAQ} \geq 1.5$ at 10 but not five years.

\section{Multivariable analyses}

Results of models fully adjusted for all covariates are shown in Table 2. Baseline RDCl was not found to be associated with increased odds of high 10-year HAQ (OR $0.94,95 \% \mathrm{Cl}: 0.73,1.22$ ). In contrast to the fiveyear model, time from onset to first appointment, being seropositive and ESR were not included. Worse deprivation was associated with increased odds of 10-year $\mathrm{HAQ} \geq 1.5$ (OR 0.79, 95\% Cl: 0.69, 0.90). Baseline HAQ remained strongly associated with high 10-year HAQ but with a smaller magnitude than in the five-year model (OR 2.47, 95\% Cl: 1.80, 3.41).

\section{HAQ sensitivity analyses}

Results are shown in Supplementary Table S4, available at Rheumatology online. There remained no association between baseline RDCl and baseline HAQ when considered binary and continuous respectively at either five $(0.03,95 \% \mathrm{Cl}:-0.06,0.12)$ or 10 years $(0.01,95 \% \mathrm{Cl}$ : $-0.17,0.18)$.

\section{Supplementary analyses: Short-Form 36 health questionnaire}

Full results of this analysis are shown in Supplementary Data S2, and model results presented in Supplementary Table S5, both available at Rheumatology online. In summary, there was no evidence of an association between RDCl at baseline and five-year SF-36, for either the physical or mental scores. 


\section{Discussion}

This study shows that baseline comorbidity, measured using the $\mathrm{RDCl}$, is not significantly associated with high $\mathrm{HAQ}$ at five or 10 years. In contrast, sociodemographic factors (increased age at disease onset, female gender and minority ethnicity) were significantly associated with higher odds of $H A Q \geq 1.5$ at five and 10 years. Worse deprivation was associated with $\mathrm{HAQ} \geq 1.5$ at 10 years.

Previous studies have shown a high comorbidity burden at diagnosis of RA, increasing over time [16, 17]. The recent work of England et al. [16] showed higher incidence and poorer trajectory of multimorbidity in patients with RA, particularly after symptom onset. Few studies, however, have examined how comorbidities are associated with RA disease outcomes in later years. Recognising the importance of comorbidities, we took the approach of adjusting for them in our models, allowing for effects of other variables to be seen, possible only because of the wealth of data within these cohorts.

In addition to functional outcome, this study considered patients likely to report poorer health-related quality of life (HRQoL) at five years, measured using the Short-Form Health Survey (SF-36) [18], as described in Supplementary Data S1, available at Rheumatology online. HAQ and SF-36 were the focus of this study, recognising the importance of these patient-reported outcomes (PROs) and the growing need to deliver more tailored, holistic patient care that takes into account the patient perspective [19]. The value of using these tools is being increasingly recognised, as focus shifts towards outcomes important to patients as well as clinicians. This study is unique in two principal ways. Firstly, in using two of the largest and longest longitudinal RA cohorts, rich in comorbidity and other clinical and sociodemographic data. Secondly, in attempting to study both biological and non-biological factors using principles of syndemic theory to provide in-depth understanding of associations between these factors and how they could impact on physical function in RA. Evidence suggests that comorbidities negatively impact on the achievement of treat-to-target goals and act as a barrier to optimal outcomes in RA [17, 20, 21]. This study goes a step further, providing evidence that sociodemographic factors potentially play an additional role, beyond comorbidities, which should not be sidelined when managing patients.

The focus of this study has been on using patient features identified at the initial rheumatology appointment to determine those more likely to have worse disease outcome in later years. A previous study [22] found that clinical data three months after onset was more informative, once treatment had begun. This work has shown that, although clinical features can aid identification of those patients for whom treatment failure is more likely, sociodemographic factors, collected at baseline, can also be helpful in guiding specific care pathways. These may relate to drug therapy and or non-drug therapeutic strategies such as more tailored physical therapy (physiotherapy) especially in view of the outcome.

The link between joint damage and physical function is well known and documented [23]. Although relevant to the HAQ outcome, radiographic data were beyond the scope of this study, which had a focus on sociodemographic and clinical factors and their potential role at baseline.

Strengths of this study include the use of large, early RA cohorts designed such that data could be collectively analysed. The long study follow-up and treatment according to best practice allow for useful, real-world information on RA treatment and outcomes. The richness of data has resulted in meaningful analyses focussing on parameters beyond those routinely reported in the literature, including sociodemographic factors. A study limitation is the age of the cohorts, partly due to previous more conservative approaches to disease management including lower use of biologic therapies, especially in the context of comorbidities. Thus, generalisability of study findings is limited. Additionally, few patients identified with a minority ethnic heritage, so their responses could not be meaningfully analysed to represent different communities. Univariable analysis showed a relationship between deprivation (IMD) and $\mathrm{HAQ} \geq 1.5$ outcome; in multivariable analysis this ceased to exist once ethnicity was included at five years. Deprivation and/or ethnicity appear to contribute to poor $\mathrm{HAQ}$ outcome, with $28 \%$ of minority ethnicity compared with $14 \%$ of white patients in the most deprived quintile within these cohorts. However, limited sampling of these subpopulations highlights an important avenue for future study to better delineate these relationships, understand these mechanisms and identify potentially modifiable factors to redress health inequalities. A further limitation is the number of hypotheses tested and lack of multiple testing correction. It is possible that some significant results are false positives, and results should be interpreted as exploratory rather than confirmatory.

In conclusion, this study found no association between baseline comorbidities and later functional outcome. However, in models adjusting for comorbidities, based on their importance and relevance on disease outcomes in RA, sociodemographic factors play a role in functional ability in the longer-term and should be considered. These findings provide ground for risk stratification in RA at first in-clinic patient review, enabling the identification of patients at higher risk of functional disability. Insights on the potential role of patientrelated, sociodemographic factors beyond traditional disease measures such as high disease activity allows for more holistic patient management and tailored intervention to be undertaken.

Funding: This work was supported by the British Medical Association (BMA) as part of the Doris Hillier Award received by E.N.

Disclosure statement: The authors have declared no conflicts of interest. 


\section{Data availability statement}

The data underlying this article will be shared on reasonable request to the corresponding author.

\section{Supplementary data}

Supplementary data are available at Rheumatology online.

\section{References}

1 Hawley DJ, Wolfe F. Pain, disability, and pain/disability relationships in seven rheumatic disorders: a study of 1,522 patients. J Rheumatol 1991;18:1552-7.

2 Maska L, Anderson J, Michaud K. Measures of functional status and quality of life in rheumatoid arthritis: health Assessment Questionnaire Disability Index (HAQ), Modified Health Assessment Questionnaire (MHAQ), Multidimensional Health Assessment Questionnaire (MDHAQ), Health Assessment. Arthritis Care Res 2011;63(Suppl 11): S4-13.

3 Conaghan PG, Hensor EMA, Keenan A-M, Morgan AW, Emery P; the YEAR Consortium. Persistently moderate DAS-28 is not benign: loss of function occurs in early RA despite step-up DMARD therapy. Rheumatology 2010; 49:1894-9.

4 Nikiphorou E, Norton S, Carpenter L et al. Secular changes in clinical features at presentation of rheumatoid arthritis: increase in comorbidity but improved inflammatory states. Arthritis Care Res 2017;69:21-7.

5 Stouten V, Westhovens R, de Cock D et al. Having a comorbidity predicts worse outcome in early rheumatoid arthritis despite intensive treatment: a post hoc evaluation of the pragmatic randomized controlled CareRA trial. Rheumatology 2021; doi: 10.1093/rheumatology/keaa841.

6 Nikiphorou E, Lempp H, Kohrt BA. Treatment failure in inflammatory arthritis: time to think about syndemics? Rheumatology 2019;58:1526-33.

7 Nikiphorou E, Carpenter L, Morris S et al. Hand and foot surgery rates in rheumatoid arthritis have declined from 1986 to 2011 , but large-joint replacement rates remain unchanged: results from two UK inception cohorts. Arthritis Rheumatol 2014;66:1081-9.

8 England BR, Sayles H, Mikuls TR, Johnson DS, Michaud $\mathrm{K}$. Validation of the rheumatic disease comorbidity index. Arthritis Care Res 2015;67:865-72.

9 Ministry of Housing Communities and Local Government. Index of multiple deprivation score, 2007. 2015. https:// data.gov.uk/dataset/5ceb7e93-bc1a-48cf-80fdfbdd15909640/index-of-multiple-deprivation-score-2007 (May 2019, last accessed).

10 Welsh index of multiple deprivation. https://gov.wales/ welsh-index-multiple-deprivation (May 2019, last accessed).
11 Northern Ireland multiple deprivation measure. https:// gov.wales/welsh-index-multiple-deprivation (May 2019, last accessed).

12 van Riel PLCM. The development of the disease activity score (DAS) and the disease activity score using 28 joint counts (DAS28). Clin Exp Rheumatol 2014;32(Suppl 85): S65-74.

13 van der Heijde DM, van 't Hof M, van Riel PL, van de PL. Development of a disease activity score based on judgment in clinical practice by rheumatologists. Rheumatology 1993;20:579-81.

14 Carpenter L, Norton S, Nikiphorou E et al. Validation of methods for converting the original Disease Activity Score (DAS) to the DAS28. Rheumatol Int 2018;38: 2297-305.

15 Nakajima A, Aoki Y, Terayama K et al. Health assessment questionnaire-disability index (HAQ-DI) score at the start of biological disease-modifying antirheumatic drug (bDMARD) therapy is associated with radiographic progression of large joint damage in patients with rheumatoid arthritis. Mod Rheumatol 2017; 27:967-72.

16 England BR, Roul P, Yang Y et al. Burden and trajectory of multimorbidity in rheumatoid arthritis: a matched cohort study from 2006 to 2015. Ann Rheum Dis 2021; 80:286-92.

17 Norton S, Koduri G, Nikiphorou E et al. A study of baseline prevalence and cumulative incidence of comorbidity and extra-articular manifestations in RA and their impact on outcome. Rheumatology 2013;52:99-110.

18 Ware JE Jr, Brook RH, Davies-Avery A et al. Conceptualization and measurement of health for adults in the health insurance study. Model of Health and Methodology. Vol. I. Report (Rand Corporation) 1980. https://www.rand.org/pubs/reports/R1987z1.html.

19 Nikiphorou E, Nurmohamed MT, Szekanecz Z. Editorial: comorbidity burden in rheumatic diseases. Front Med 2018;5:197.

20 Nikiphorou E, Norton S, Young A et al. The association of obesity with disease activity, functional ability and quality of life in early rheumatoid arthritis: data from the Early Rheumatoid Arthritis Study/Early Rheumatoid Arthritis Network UK prospective cohorts. Rheumatology 2018;57:1194-202.

21 Radner H, Smolen JS, Aletaha D. Impact of comorbidity on physical function in patients with rheumatoid arthritis. Ann Rheum Dis 2010;69:536-41.

22 Fanouriakis A, Papalopoulos I, Gergianaki I et al. In early arthritis patients, high HAQ at baseline and DAS28 at three months predict suboptimal outcomes at two years: a retrospective cohort study. Clin Exp Rheumatol 2018; 36:806-13.

23 Nikiphorou E, Norton SJ, Carpenter L et al. Remission vs low disease activity: function, quality of life and structural outcomes in the Early Rheumatoid Arthritis Study and Network. Rheumatology 2020;59:1272-80. 